\title{
ESTUDO EXPLORATÓRIO DE INSPEÇÃO DE MANIFESTAÇÕES PATOLÓGICAS DE FACHADAS UTILIZANDO VEÍCULO AÉREO NÃO TRIPULADO (VANT)
}

\section{EXPLORATORY STUDY INSPECTION OF PATHOLOGICAL MANIFESTATIONS OF FACADE USING UNMANNED AERIAL VEHICLE (UAV)}

\author{
MOURA JR., JOSÉ MARIA DE \\ Estudante de Engenharia Civil \\ Universidade de Pernambuco \\ Pernambuco; Brasil \\ jmdemjr@gmail.com
}

\author{
LORDSLEEM JR., ALBERTO CASADO \\ Engenheiro Civil \\ Universidade de Pernambuco \\ Pernambuco; Brasil \\ acasado@poli.br
}

\author{
RUIZ, RAMIRO DANIEL BALLESTEROS \\ Engenheiro Civil \\ Universidade de Pernambuco \\ Pernambuco; Brasil \\ ramiro.dbr@hotmail.com
}

\section{RESUMO}

A construção civil está em constante evolução, sempre buscando a inserção de novas tecnologias. Uma destas tecnologias é o Veículo Aéreo Não Tripulado (VANT). Este artigo tem como principal objetivo realizar estudo exploratório do uso de VANT como ferramenta visual para inspeção de manifestações patológicas de fachadas de edifícios. A revisão bibliográfica foi baseada no modelo PRISMA (Preferred Reporting Items for Systematic reviews and Meta-Analysis), cuja buscas foram realizadas na plataforma Portal de Periódicos CAPES/MEC. A pesquisa exploratória de campo foi realizada para caracterizar o panorama atual das empresas de drone na Região Metropolitana do Recife - RMR/PE, por meio de questionários e entrevistas. A partir dos resultados foi possível identificar padrões relacionados ao planejamento de voo e a classificação dos drones. Além disso, também foram realizados voos no Instituto de Inovação Tecnológica (IIT), objetivando aplicar os protocolos das atividades de captura de imagens para o processamento e a análise de dados da fachada da edificação. Os resultados revelaram que é possível a identificação de patologias em fachadas com o uso do drone e que o processo é mais seguro e veloz comparado com os métodos convencionais de inspeção, como as cadeiras suspensas em cabo de aço.

Palavras-chave: manifestação patológica, fachada, veículo aéreo não tripulado (VANT).

\begin{abstract}
Civil construction is constantly evolving, always trying to insert new Technologies, such as the Unmanned Aerial Vehicle (UAV). The main objective of this research project is to conduct an exploratory study of the use of UAV as a visual tool to inspect pathological manifestations in building facades. The literature review was based on the PRISMA (Preferred Reporting Items for Systematic reviews and Meta-Analysis) model, whose searches were performed on the CAPES / MEC Portal of Periodicals. The exploratory field research was conducted to characterize the current panorama of drone companies in the Metropolitan Region of Recife - RMR / PE, through questionnaires and interviews. From the results, it was possible to identify patterns related to flight planning and drone classification. Also, flights were made at the Institute of Technological Innovation (IIT), aiming to apply the protocols 2 of the image capture activities for the processing and analysis of the building façade data. The results revealed that it is possible to identify façade pathologies with the use of a drone and that the process is safer and faster compared to conventional inspection methods such as suspended scaffolds.
\end{abstract}

Keywords: pathological manifestation, façade, unmanned aerial vehicle (UAV). 


\section{INTRODUÇÃO}

Num cenário de marcantes desafios na construção de edifícios, onde é imperiosa a necessidade de melhoria da produtividade, qualidade, desempenho e, preponderante, a redução de custos, é importante a preservação/recuperação de edificações existentes (PINHO, 2013; CONSTRUBUSINESS, 2016; LIMA; GOMES, 2017).

O aparecimento cada vez mais precoce de manifestações patológicas está aliado ao crescente grau de insatisfação dos clientes, apontados pelas instituições de proteção e defesa dos consumidores (CAMPOS, 2011; PROCON, 2012; ALVES, 2016).

Um dos grandes problemas relatados por construtoras e moradores é o aparecimento de manifestações patológicas nas construções. A ocorrência de manifestações patológicas em edificações é resultante, em grande parte: pela adoção de procedimentos de execução inadequados, pelo não atendimento das recomendações da normalização e falhas nas especificações de projeto e dos materiais (THOMAZ, 1989; COSTA E SILVA, 2008; RIBEIRO, 2014).

Segundo Figueiredo Júnior (2017, p.12) "As patologias nas edificações, na maioria das vezes, não ocorrem apenas por um fator, mas por uma combinação destes e traz prejuízos para todos os membros da cadeia produtiva da construção envolvidos, além de causar grande impacto na estética da edificação como um todo e, em algumas circunstâncias, ocasionar graves acidentes."

Particularmente, as manifestações patológicas das fachadas assumem destacada importância em função do risco à segurança e a perda de desempenho do edifício, já que respondem também pelos aspectos relativos ao conforto, à higiene e à saúde (CEOTTO et al., 2005; COSTA E SILVA, 2008).

As principais manifestações patológicas incidentes nas fachadas são comumente associadas ao descolamento do revestimento, fissuras/trincas e umidade (LORDSLEEM JR., 1997; COSTA E SILVA, 2001; SAHADE, 2005).

Sobre o assunto, Lichtenstein (1985) recomenda que a resolução das manifestações patológicas deve ser orientada por procedimento de atuação que geralmente envolve as seguintes etapas: inspeção, anamnese, exames, diagnóstico, prognóstico e terapia a ser adotada.

Em especial, merece uma destacada atenção na fachada, a realização da etapa de inspeção (vistoria para verificar as condições de desempenho e determinar medidas preventivas e corretivas que se fizerem necessárias), em virtude da inerente complexidade (altura, tamanho, dificuldades de acesso e condições exposição).

Para os propósitos deste artigo, busca-se associar a realização da inspeção de fachadas com o emprego de drone ou veículo aéreo não tripulado (VANT), em inglês Unmanned Aerial Veicheles (UAV), com o intuito de verificar a viabilidade de sua utilização, visto que a mesma ainda é pouco difundida no Brasil.

\section{REFERENCIAL TEÓRICO}

A Agência Nacional de Aviação Civil (ANAC) define VANT ou drone como sendo "Aeronave projetada para operar sem piloto a bordo e que não seja utilizada para fins meramente recreativos" (ANAC, 2012, pág. 3).

O Veículo Aéreo Não Tripulado (VANT), em inglês Unmanned Aerial Vehicle/System (UAV/UAS), também conhecido como "drone", é qualquer tipo de aeronave que não precisa de pilotos embarcados para ser guiada, sendo controlados a distância por meios eletrônicos e computacionais, com ou sem a supervisão e pilotagem de humanos e que não seja usada para fins meramente recreativos.

Inicialmente, os VANT foram desenvolvidos para operações militares durante os anos 1970 e 1980. Recentemente, no entanto, surgiu uma nova geração de aeronaves multirotor, que utiliza lâminas de passo fixo para controlar o movimento do veículo, variando a velocidade relativa de cada rotor para alterar o impulso e o torque produzidos, permitindo uma amplitude de movimento única.

Segundo Nascimento (2014, p. 12) “Atualmente suas aplicações não militares são para diversas finalidades, como por exemplo: fotogrametria, monitoramento de reservas ambientais, do clima, no jornalismo, fazer entregas rápidas, e 
outros. No Brasil, o destaque está na agricultura, com utilizações para verificação de irrigação de plantações, aplicação de fertilizantes e até mesmo no controle de formação de formigueiros em plantações de eucaliptos.”

Atualmente suas aplicações são diversas: agricultura, fotogrametria, monitoramento de reservas ambientais e do clima, no jornalismo, fazer entregas rápidas, entre outras. Apenas recentemente foi publicada a legislação brasileira que regula os requisitos gerais para as aeronaves não tripuladas de uso civil (ANAC, 2017).

Na construção civil, o seu uso ainda é limitado (COSTA; MELO, 2015), embora perceba-se que o VANT pode ser utilizado de diversas maneiras, e com o desenvolvimento cada vez maior da tecnologia aliada a outros tipos de câmeras e sensores, permite o acesso a locais antes inacessíveis ao ser humano e a outras tecnologias (NASCIMENTO, 2014; EMELIANOV et al., 2014; KIM et al., 2016).

Álvares et al. (2016) também destacam o fato da tecnologia ser recente na construção civil, ao discutir o desenvolvimento de modelos 3D a partir de imagens coletadas com os VANTs para atividades relacionadas ao gerenciamento das construções.

Por outro lado, diversos autores (EMELIANOV et al., 2014; NASCIMENTO, 2014; COSTA; MELO, 2015; KIM et al., 2016) enfatizam as vantagens da utilização dos VANTs, notadamente pelo aumento da velocidade e expansão da cobertura de realização das atividades, além da redução do risco de segurança das pessoas.

Segundo Siebert e Teizer (2014, apud Costa e Melo, 2015, p. 3), "o avanço dos VANTs se dá devido ao baixo custo, agilidade de coleta e processamento, facilidade de manobra das aeronaves, e ao eficiente sistema de captura de imagem. Atualmente no mercado existem dois tipos de VANTs, a diferença entre eles é basicamente o sistema aerodinâmico, podendo ser em asa fixa ou rotativa"

\section{METODOLOGIA}

Para etapa de desenvolvimento inicial da pesquisa, procedeu-se a seleção de bibliografia de referência para o necessário embasamento teórico dos conceitos relativos ao tema. Para isso, foi utilizado o modelo PRISMA, Preferred Reporting Items for Systematic reviews and Meta-Analyses. Além disso, foi realizado uma pesquisa de campo exploratória com objetido de fazer uma caracterização das empresas que trabalham com drone nas cidades de Recife e Olinda, Pernambuco. Uma pesquisa de campo experimental foi feita para análise de inspeção de manifestações patológicas de um prédio em Recife, Pernambuco.

\subsection{Etapa 1: Modelo PRISMA}

Os artigos selecionados para estudo foram encontrados no portal de periódicos CAPES (Coordenação De Aperfeiçoamento De Pessoal De Nível Superior). A pesquisa foi realizada em três idiomas: inglês, português e espanhol. As palavras chave usadas para a busca estão descritas na tabela 1. A busca avançada foi realizada no "assunto" e no "título". Os filtros "Por pares", "Por ano", "Por idioma" e "Por tópico" foram aplicados para a seleção dos artigos.

Tabela 1 - Palavras chave utilizadas em cada idioma

\begin{tabular}{c|c}
\hline IDIOMAS & PALAVRAS CHAVE \\
\hline Português & $\begin{array}{c}\text { Inspeção Predial, Fachada, Manifestação Patológica, Veículo Aéreo Não Tripulado } \\
\text { (VANT), Mapeamento da Fachada, Patologia de fachada, Fissuras de fachada, Trincas de } \\
\text { fachada, Umidade em fachada, Revestimento de Fachada }\end{array}$ \\
\hline Inglês & $\begin{array}{c}\text { Building Inspection, Facade, Pathological Manifestation, Unmanned Aerial Vehicles } \\
\text { (UAV), Facade Mapping, Pathology of facade, Facade cracks, Facade humidity, Facade } \\
\text { Coating }\end{array}$ \\
\hline Espanhol & $\begin{array}{c}\text { Inspección de fachada, Fachada, Manifestación Patológica, Vehículo Aéreo No } \\
\text { Tripulado (VANT), Mapeamiento de Fachada, Patología de fachada, Fisuras en fachada, } \\
\text { Grietas en fachada, Humedad en la fachada, Revestimiento de Fachada }\end{array}$ \\
\hline
\end{tabular}


Durante a revisão sistemática, foram considerados elegíveis os estudos: acessíveis no portal de periódicos CAPES para download gratuito; por pares (trabalhos científicos submetidos ao escrutínio de um ou mais especialistas do mesmo escalão que o autor); por ano; por idioma (trabalhos em português, inglês e espanhol); por tópico (artigos relacionadas à engenharia civil, arquitetura e construção); por duplicatas (trabalhos científicos não duplicados); por título; por resumo.

\subsection{Etapa 2: Pesquisa de campo exploratória}

Para a realização das entrevistas, foi desenvolvido um questionário estruturado em quatro partes: dados da empresa; dados do responsável, drone e planejamento de voo. Os dados que compuseram o questionaram foram obtidos através da literatura.

No tópico relacionado a empresa, foram coletados os seguintes dados: nome, endereço, fone, e-mail, área de atuação, tempo de atuação, número de obras e certificações. Para a parte relacionada ao responsável: nome, cargo, fone, e-mail, formação, capacitação para utilização de drone e verificação de licença para uso do drone. Já para a parte relacionada ao drone, primeiro verificou-se qual o modelo utilizado pela empresa, em seguida suas classificações quanto ao: uso, tipos de asas, peso, alcance/bateria e tipo de câmera utilizado. No planejamento de voo foi verificado se era analisado: locais alternativos para pouso e decolagem, as condições meteorológicas antes do voo, utilização baterias extras, transferência de arquivos a cada pouso, analise de topografia do local, checklist de equipamentos, altitude do voo, perímetro do local, região a ser mapeada, velocidade do voo, direção do vento, índice de sobreposição de imagens. Foi analisado se era utilizado algum software para preenchimento de dados ou se era feito o preenchimento de maneira manual. As entrevistas tiveram como objetivo fazer uma caracterização das empresas que trabalham com drone em Recife e Olinda.

A amostragem de empresas foi definida em pesquisa na internet e em função do interesse da empresa na participação. No total foram realizadas 6 entrevistas com empresas ligadas ao ramo da fotografia, fotogrametria, levantamento topográfico e monitoramento de obras.

\subsection{Etapa 3: Pesquisa de campo experimental}

Para o desenvolvimento do presente trabalho foram utilizados os seguintes equipamentos:

- Drone DJI Phantom 4 PRO Plus (figura 1);

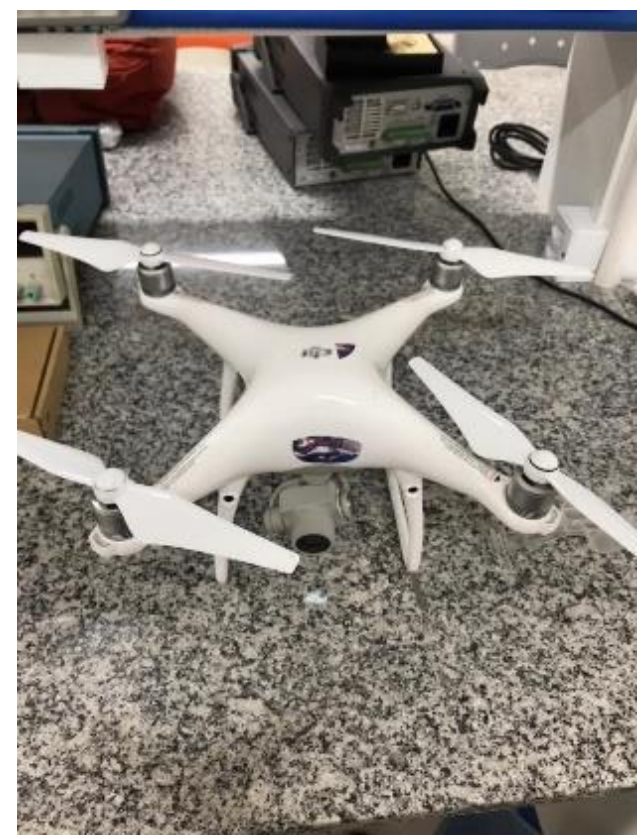

Figura 1: Drone DJI Phantom 4 PRO Plus

O VANT utilizado é do tipo quadricóptero, modelo DJI Phantom 4 PRO Plus, com autonomia máxima de 28 minutos de voo por bateria; operado por controle remoto, câmera original do drone acoplada, com resolução FULL HD e sistema GPS, permitindo a captura de imagens georreferenciadas. 
A metodologia utilizada baseou-se no estudo de imagens aéreas obtidas através da câmera original acoplada ao drone, possibilitando à observação de anomalias já existentes, tendo em vista que as fotos são tiradas em alta resolução.

- Software Agisoft PhotoScan.

O software utilizado para modelagem 3D foi o Agisoft PhotoScan (figura 2). O mesmo realiza a conversão de imagens 2D georreferenciadas, por meio da geração automática de nuvem de pontos e texturização de modelos 3D. O software também possui outras ferramentas, como a de medição, que possibilita levantar distâncias, áreas e volumes a partir dos modelos 3D.

O software desenvolve o mapeamento 3D inicialmente por meio da compilação do conjunto de imagens e extração de pontos chave (key-points), foto a foto. Em seguida ocorre a combinação e marcação dos key-points entre as imagens, gerando uma nuvem de pontos 3D. Após este processamento inicial, é feita a densificação e filtragem dos pontos. A partir dessa nuvem densificada é possível então realizar a texturização de uma malha 3D, por meio da triangulação dos pontos, gerando assim o modelo 3D da superfície registrada nas imagens.

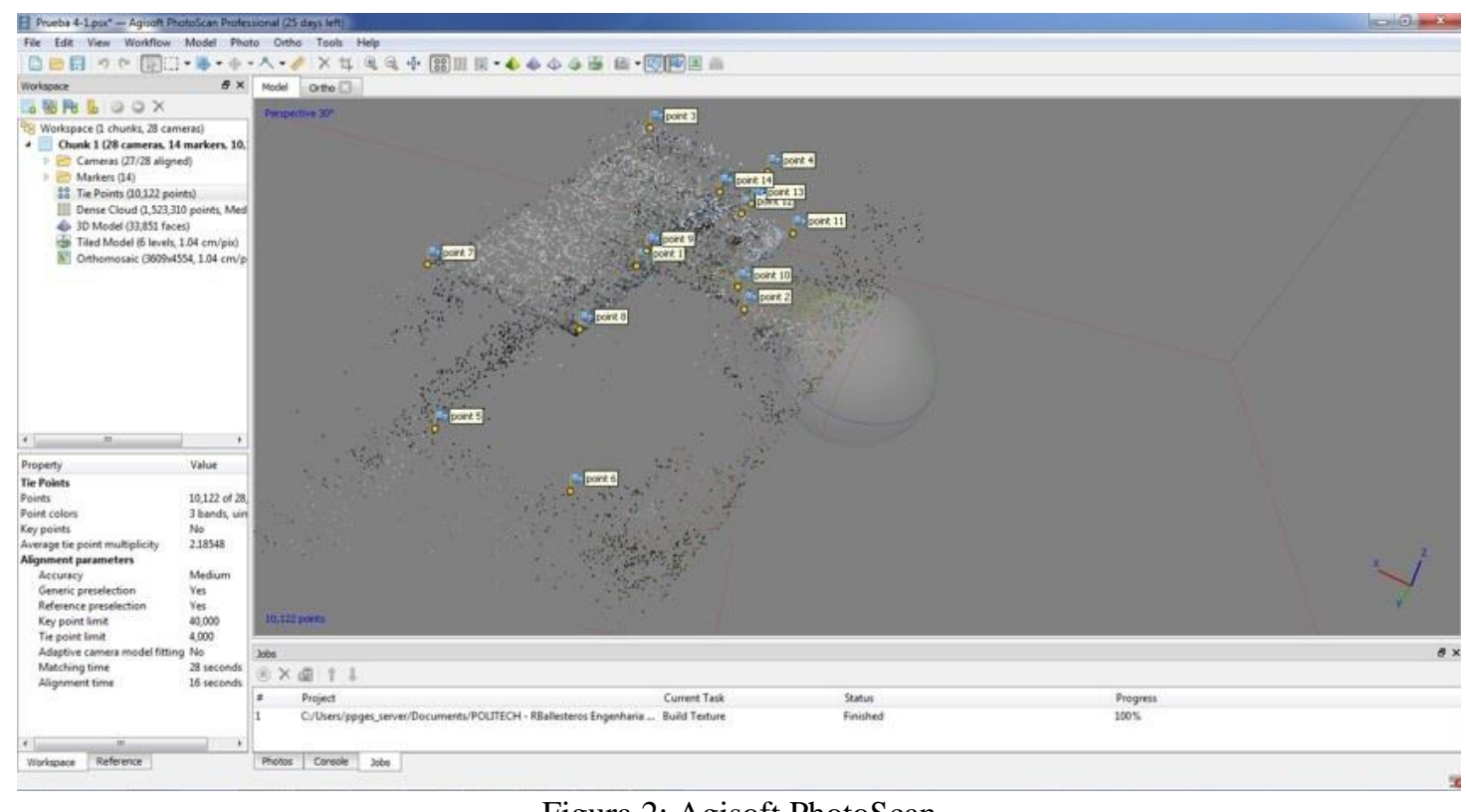

Figura 2: Agisoft PhotoScan

O estudo foi realizado no Instituto de Inovação Tecnológica (figura 3), localizado no Parqtel, na cidade de Recife, Pernambuco. O local permitiu a utilização do drone com segurança pois não existe prédios próximos, além disso, era um local de fácil acesso para a pesquisa.

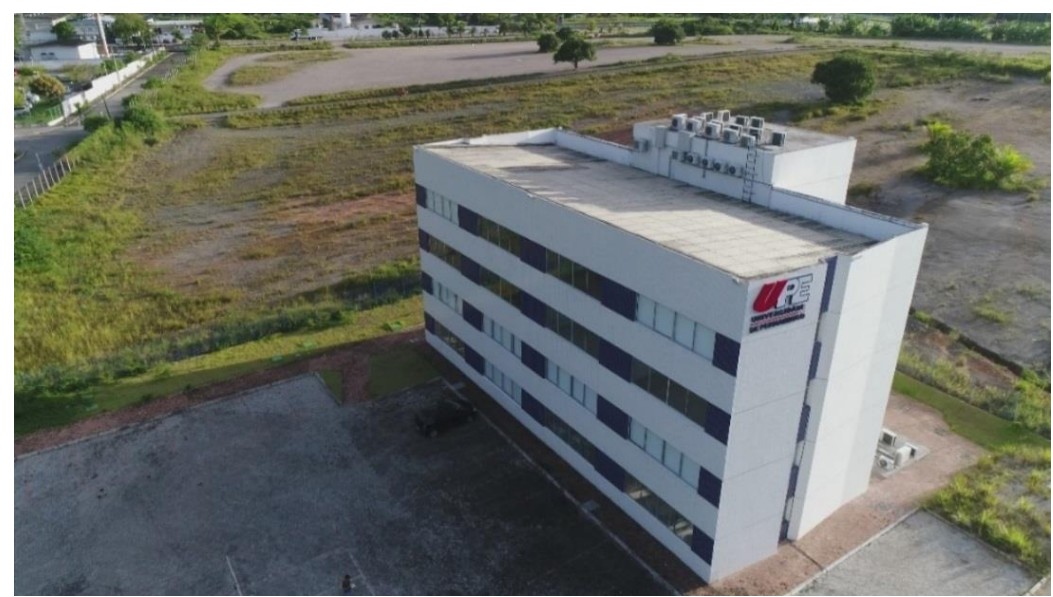

Figura 3: Instituto de Inovação Tecnológica 


\section{RESULTADOS E DISCUSSÃO}

A pesquisa foi realizada através de uma pesquisa bibliográfica com a aplicação da metologia PRISMA. Em seguida foi feita uma investigação por coleta de dados junto a um total de 6 (SEIS) empresas que trabalham com drone nas cidades de Recife e Olinda, Pernambuco. E, por último, realizou-se uma coleta de campo em uma edificação.

A análise dos dados coletados foi subdividida em seis etapas distintas, conforme a figura 4:

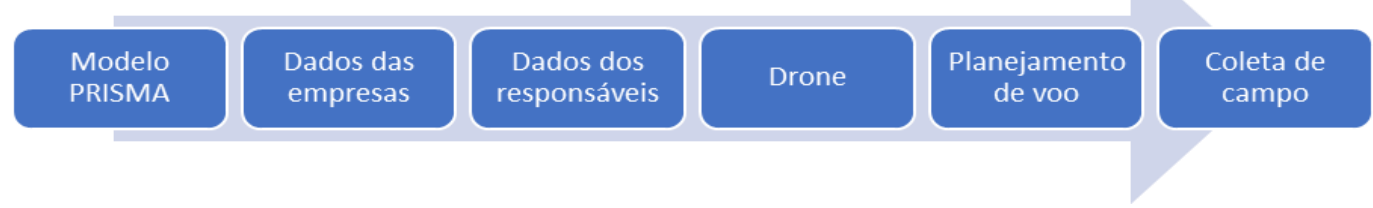

Figura 4: Fluxograma das etapas do projeto

\subsection{Modelo PRISMA}

Através da aplicação da metodologia PRISMA foram encontrados 10949 artigos no portal de periódicos CAPES, sendo um total de 219 em português, 1044 em espanhol e 9686 em inglês.

Conforme a aplicação dos critérios de elegibilidade para a inclusão e exclusão dos estudos, foram excluídos 6472 por pares, 2809 por ano, 58 por idioma, 1290 por tópico, 276 estudos pelo título e 10 pelo resumo (abstract), resultando em 5 textos. Também foram encontrados e excluídos estudos de revisão sistemática, meta-análises, sem o texto completo disponível e com abstract não disponível.

A Figura 5 apresenta a estratégia de busca utilizada em cada base de dados e os resultados obtidos.

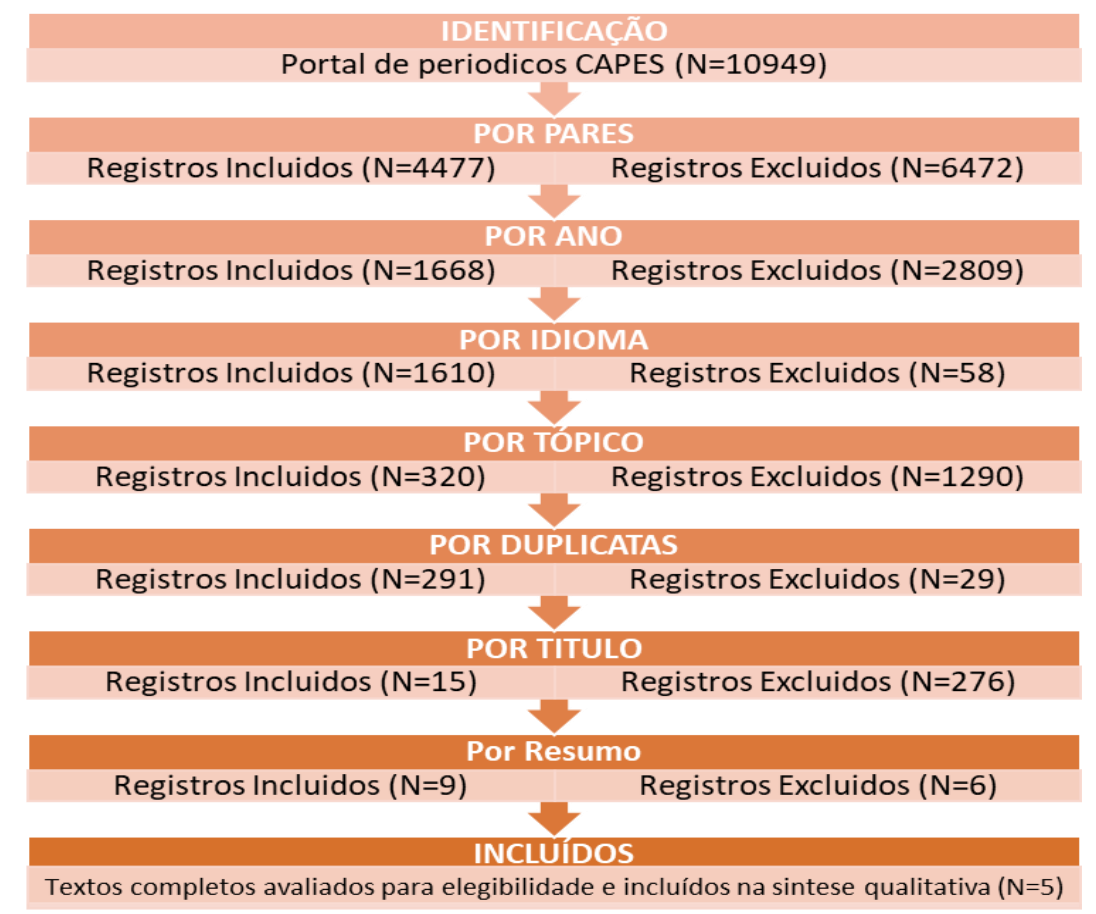

Figura 5: Fluxograma baseado no modelo PRISMA com os resultados da seleção dos artigos

Após a elaboração da revisão sistemática, observou-se a existência de diferentes tipos de VANT úteis para o propósito da inspeção visual na indústria da construção civil. Os VANT de asas rotatórias são os mais simples de pilotar, pois a sustentação é dada pelo conjunto de motores controlados por um sistema eletrônico sofisticado que geralmente garante uma boa estabilidade da aeronave, sendo sua decolagem e aterragem vertical. Portanto, é a melhor opção para carregar câmeras e outros objetos sem grandes deslocamentos do ponto de decolagem. 


\subsection{Dados das empresas}

A tabela 2 relaciona as respostas identificadas como principais para os dados das empresas de drone participantes da pesquisa.

Tabela 2 - Dados das empresas

\begin{tabular}{|c|c|c|c|c|c|c|}
\hline \multirow{2}{*}{ CARACTERIZAÇÄO } & \multicolumn{6}{|c|}{ EMPRESAS } \\
\hline & $\mathrm{A}$ & B & C & $\mathrm{D}$ & $E$ & $\mathbf{F}$ \\
\hline ÁREA DE A TUAÇÃO & Construção civil & $\begin{array}{l}\text { Acompanhamento } \\
\text { de obra e publicidade }\end{array}$ & $\begin{array}{c}\text { Imagens aéreas e } \\
\text { levantamento } \\
\text { topográfico }\end{array}$ & $\begin{array}{c}\text { Fotografia e filmagem } \\
\text { aérea }\end{array}$ & Engenharia & $\begin{array}{c}\text { Filmagem e fotografia } \\
\text { aérea }\end{array}$ \\
\hline DRONE & DJI Inspire $1 \mathrm{v} 2.0$ & $\begin{array}{c}\text { DJI Inspire } 2 \text { v2.0 DJI } \\
\text { Phantom } 4 \text { Pro }\end{array}$ & $\begin{array}{c}\text { DJI Phantom } 4 \text { Pro } \\
\text { Octacoptero } 8 R\end{array}$ & DJI Mavic Pro & DJI Phantom 4 Pro & DJl Phantom 4 Pro \\
\hline TEMPO DE ATUAÇÄO & 3 meses & 5 anos & 2 anos & 1 ano e 6 meses & 12 anos & 3 anos \\
\hline $\begin{array}{l}\mathrm{N}^{0} \text { DE PROJETOS } \\
\text { RELACIONADOS A } \\
\text { ENGENHARIA }\end{array}$ & 1 & 50 & 40 & 8 & 500 (aproximado) & 5 \\
\hline $\begin{array}{c}\text { QUAL TIPO } \\
\text { DE TRABALHO? }\end{array}$ & $\begin{array}{l}\text { Ensaios técnicos; } \\
\text { Avaliações de bens e } \\
\text { imóveis; Fiscalização } \\
\text { de obras; Inspeção } \\
\text { predial com drone }\end{array}$ & $\begin{array}{c}\text { Acompanhamento de } \\
\text { obra }\end{array}$ & $\begin{array}{l}\text { Levantamentos } \\
\text { topográficos }\end{array}$ & $\begin{array}{c}\text { Inspeção e } \\
\text { Monitoramento de } \\
\text { obras; time-lapse }\end{array}$ & Consultoria & $\begin{array}{l}\text { Levantamento } \\
\text { topografico }\end{array}$ \\
\hline
\end{tabular}

Foi possível identificar que o modelo mais utilizado entre as empresas é o DJI Phantom 4 Pro. O tempo de atuação variou bastante, com empresas relativamente novas e empresas já consolidadas no mercado. O número de projeto variou muito, devido, também, a idade das empresas, onde, a empresa A tem uma média de 4 projetos/ano (visto que a empresa só possui 1 projeto feito e tem 3 meses de atuação no mercado), a empresa $\mathrm{B}$ tem 10 projetos/ano, empresa $\mathrm{C}$ tem 20 projetos/ano, empresa $\mathrm{D}$ possui uma média de 5,33 projetos/ano, empresa $\mathrm{E}$ tem 41,67 projetos/ano e a empresa $\mathrm{F}$ tem 1,67 projetos/anos.

Dos resultados obtidos na pesquisa, também foi possível identificar que todas as empresas trabalham com algo relacionado a engenharia civil, mostrando que o drone é uma ferramenta útil para utilização nesse campo.

\subsection{Dados dos responsáveis}

A tabela 3 relaciona as respostas identificadas como principais para os dados dos responsáveis participantes da pesquisa.

Tabela 3 - Dados dos responsáveis

\begin{tabular}{c|c|c|c|c|c|c}
\hline \multirow{2}{*}{ CARACTERIZAÇÄO } & \multicolumn{4}{|c}{ EMPRESAS } \\
\cline { 2 - 6 } & A & B & C & D & E \\
\hline \multirow{2}{*}{ FORMAÇÄO } & $\begin{array}{c}\text { Graduado em } \\
\text { Engenharia }\end{array}$ & $\begin{array}{c}\text { Licenciatura em } \\
\text { Administração }\end{array}$ & $\begin{array}{c}\text { Engenharia } \\
\text { Mecânica }\end{array}$ & \multirow{2}{*}{ Tecnólogo } & $\begin{array}{c}\text { Técnico em } \\
\text { Construção Civil }\end{array}$ & $\begin{array}{c}\text { Técnico de } \\
\text { Programação }\end{array}$ \\
\hline CAPACITAÇÄO & Não & Não & Não & Sim & Não & Sim \\
\hline LICENÇA & Sim & Sim & Não & Sim & Não \\
\hline
\end{tabular}

Apenas 33,33\% fizeram algum curso de capacitação para utilização do drone. Os responsáveis alegaram que a utilização do equipamento é simples e não sentiram necessidade de fazer algum curso relacionado. Também foi possível identificar que $66,67 \%$ possuem licença para utilização do drone.

\subsection{Drone}

A tabela 4 relaciona as respostas identificadas como principais para os dados relacionados ao drone. 
Tabela 4 - Dados do drone

\begin{tabular}{|c|c|c|c|c|c|c|}
\hline \multirow{2}{*}{ CARACTERIZA ÇÃO } & \multicolumn{6}{|c|}{ DRONE } \\
\hline & $A$ & B & $\mathrm{C}$ & D & $E$ & $F$ \\
\hline $\begin{array}{l}\text { CLASSIFICAÇÃO } \\
\text { QUANTO AS ASAS }\end{array}$ & Rotatórias & Rotatórias & Rotatórias & Rotatórias & Rotatórias & Rotatórias \\
\hline $\begin{array}{c}\text { CLASSIFICAÇÃO DE } \\
\text { ACORDO COM O } \\
\text { PESO }\end{array}$ & $\begin{array}{c}\text { Classe } 3 \text { (Abaixo } \\
\text { de } 25 \mathrm{~kg})\end{array}$ & $\begin{array}{c}\text { Classe } 3 \text { (Abaixo } \\
\text { de } 25 \mathrm{~kg})\end{array}$ & $\begin{array}{c}\text { Classe } 3 \text { (Abaixo } \\
\text { de } 25 \mathrm{~kg})\end{array}$ & $\begin{array}{c}\text { Classe } 3 \text { (Abaixo } \\
\text { de } 25 \mathrm{~kg})\end{array}$ & $\begin{array}{c}\text { Classe } 3 \text { (Abaixo } \\
\text { de } 25 \mathrm{~kg} \text { ) }\end{array}$ & $\begin{array}{c}\text { Classe } 3 \text { (Abaixo } \\
\text { de } 25 \mathrm{~kg})\end{array}$ \\
\hline $\begin{array}{c}\text { QUAL A } \\
\text { CLASSIFICAÇÄO } \\
\text { QUANTO AO } \\
\text { ALCANCE/BA TERIA? }\end{array}$ & $\begin{array}{l}\text { Low cost close } \\
\text { range (Alcance 0- } \\
5 \mathrm{Km} \text { e Bateria 20- } \\
60 \text { minutos) }\end{array}$ & $\begin{array}{l}\text { Low cost close } \\
\text { range (Alcance 0- } \\
5 \mathrm{Km} \text { e Bateria 20- } \\
60 \text { minutos) }\end{array}$ & $\begin{array}{l}\text { Low cost close } \\
\text { range (Alcance 0- } \\
5 \mathrm{Km} \text { e Bateria 20- } \\
60 \text { minutos) }\end{array}$ & $\begin{array}{l}\text { Low cost close } \\
\text { range (Alcance 0- } \\
5 \mathrm{Km} \text { e Bateria 20- } \\
60 \text { minutos) }\end{array}$ & $\begin{array}{l}\text { Low cost close } \\
\text { range (Alcance 0- } \\
5 \mathrm{Km} \text { e Bateria 20- } \\
60 \text { minutos) }\end{array}$ & $\begin{array}{l}\text { Low cost close } \\
\text { range (Alcance 0- } \\
5 \mathrm{Km} \text { e Bateria 20- } \\
60 \text { minutos) }\end{array}$ \\
\hline $\begin{array}{c}\text { QUAL A } \\
\text { CLASSIFICAÇÄO } \\
\text { QUANTO AO TIPO } \\
\text { DE CAMERA? }\end{array}$ & $\begin{array}{l}\text { Câmera Aérea: Dji } \\
\text { Zenmuse X3 12MP }\end{array}$ & $\begin{array}{l}\text { Câmera Aérea: } \\
\text { Incorporada }\end{array}$ & $\begin{array}{l}\text { Câmera Aérea e } \\
\text { Termográfica }\end{array}$ & $\begin{array}{l}\text { Câmera Aérea: } \\
\text { Incorporada }\end{array}$ & $\begin{array}{l}\text { Câmera Aérea: } \\
\text { Incorporada }\end{array}$ & $\begin{array}{l}\text { Câmera Aérea: } \\
\text { Incorporada }\end{array}$ \\
\hline
\end{tabular}

Todos os drones utilizados entre as empresas estão na classificação de asas rotatórias, que são aqueles que usam rotores para levantar voo; classe 3 segundo a ANAC, que são aqueles abaixo de $25 \mathrm{~kg}$; e classe Low Cost Close Range, que são aqueles com alcance entre 0 e 50 quilômetros e bateria entre 20 e 60 minutos.

Em relação a classificação quanto ao tipo de câmera, 83,33\% utilizam a câmera original do drone, enquanto que uma das empresas optou por fazer a troca do equipamento para utilização em trabalho especifico.

\subsection{Planejamento de voo}

A tabela 5 relaciona as respostas identificadas como principais para os dados relacionados ao planejamento de voo.

Tabela 5 - Dados do planejamento de voo

\begin{tabular}{|c|c|c|c|c|c|c|}
\hline \multirow{2}{*}{ CARACTERIZAÇÄO } & \multicolumn{6}{|c|}{ PLANEJA MENTO DO VOO } \\
\hline & A & B & $\mathrm{C}$ & $\mathbf{D}$ & E & $\mathbf{F}$ \\
\hline $\begin{array}{l}\text { UTILIZA ALGUM } \\
\text { SOFTWARE? }\end{array}$ & Não & Não & $\begin{array}{c}\text { Sim } \\
\text { Map Pilot }\end{array}$ & $\begin{array}{c}\text { Sim } \\
\text { Google Maps }\end{array}$ & Não & $\begin{array}{c}\text { Sim } \\
\text { DroneDeploy }\end{array}$ \\
\hline $\begin{array}{c}\text { ANALISA LOCAIS } \\
\text { ALTERNA TIVOS PARA } \\
\text { POUSO E DECOLA GEM? }\end{array}$ & Sim & Não & Sim & Sim & Sim & Sim \\
\hline $\begin{array}{c}\text { VERIFICA A S CONDIÇÕES } \\
\text { METEOROLOGICA S ANTES } \\
\text { DO VOO? }\end{array}$ & Sim & Sim & Sim & Sim & Sim & Sim \\
\hline $\begin{array}{l}\text { LEVA BA TERIA S } \\
\text { EXTRAS? }\end{array}$ & Sim & Sim & Sim & Sim & Sim & Sim \\
\hline $\begin{array}{c}\text { TRANSFERE OS ARQUIVOS } \\
\text { A CADA POUSO? }\end{array}$ & Sim & Não & Não & Não & Não & Sim \\
\hline $\begin{array}{c}\text { ANALISA A } \\
\text { TOPOGRAFIA DO LOCAL? }\end{array}$ & Não & Não & Sim & Sim & Não & Sim \\
\hline $\begin{array}{l}\text { FAZ CHECKLIST DOS } \\
\text { EQUIPAMENTOS? }\end{array}$ & Sim & Sim & Sim & Sim & Não & Sim \\
\hline $\begin{array}{l}\text { REGIÄO A SER } \\
\text { MAPEADA }\end{array}$ & Sim & Sim & Sim & Sim & Não & Sim \\
\hline PERÍMETRO DO LOCAL & Sim & Sim & Sim & Sim & Não & Sim \\
\hline $\begin{array}{l}\text { LOCAL DE LANÇAMENTO E } \\
\text { POUSO }\end{array}$ & Sim & Não & Sim & Sim & Sim & Sim \\
\hline DIREÇÄO DO VENTO & Sim & Sim & Sim & Sim & Não & Sim \\
\hline ALTITUDE & Sim & Sim & Sim & Sim & Não & Sim \\
\hline VELOCIDADE DO VOO & $\begin{array}{c}\text { Sim } \\
\text { Obs: velocidade } \\
\text { baixa }(3 \mathrm{~km} / \mathrm{h})\end{array}$ & Sim & Sim & Sim & Não & $\begin{array}{c}\text { Sim } \\
\text { Obs: velocidade } \\
\text { baixa }(15 \mathrm{~m} / \mathrm{s})\end{array}$ \\
\hline $\begin{array}{c}\text { ÍNDICE DE } \\
\text { SOBREPOSIÇÄO DE } \\
\text { IMAGENS }\end{array}$ & Não & Não & Sim & Sim & Não & Sim \\
\hline
\end{tabular}

Os dados utilizados para a parte de planejamento de voo foram retirados da literatura e de aplicativos de drone utilizados para planejamento de voo. Foram analisados diversos parâmetros, entre eles: verificação de condições meteorológicas antes do voo, checklist de equipamentos, uso de baterias extras, velocidade do voo e índice de sobreposição de imagens. Os dados critérios estão representados graficamente na figura 6. 


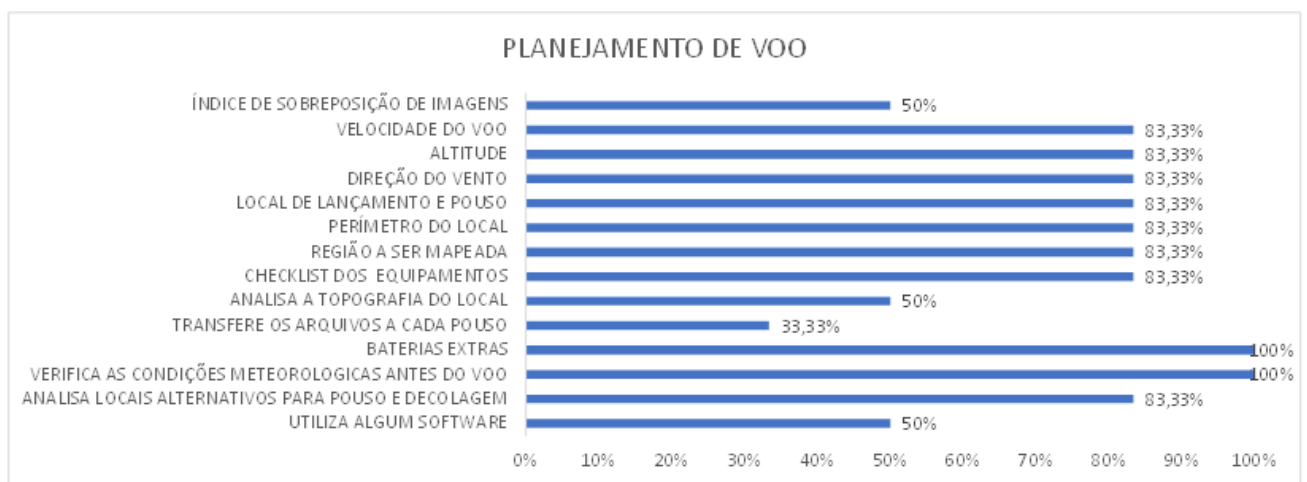

Figura 6: Gráfico de planejamento de voo

Os resultados mostram que $83,33 \%$ das empresas entrevistadas analisam a velocidade do voo, altitude, direção do vento, local de lançamento de pouso, perímetro do local, região a ser mapeada e faz checklist de equipamentos. Apenas $33,33 \%$ faz transferência de arquivos a cada pouso, esse número pequeno se dá porque algumas empresas utilizarem cartão de memória com muito espaço e alguns trabalhos específicos não necessitam de muitas imagens e vídeos. Além disso, 50\% analisa o índice de sobreposição de imagens e a topografia do local e $100 \%$ das empresas verificam as condições meteorológicas antes do voo e levam baterias extras. Entre as empresas analisadas, $50 \%$ utilizam algum software para preenchimento do planejamento de voo e $50 \%$ prefere fazer de maneira manual.

\subsection{Coleta de Campo}

Foram realizadas um total de 4 visitas de campo para coleta de dados. A tabela 6 detalha a quantidade de voos feitos para cada visita de campo, o horário, local, número de fotos, distancia, altitude e duração do voo. Somando todos os voos obteve-se um total de 469 imagens.

Tabela 6 - Planilha Flight Log

\begin{tabular}{c|c|c|c|c|c|c|c|c}
\hline № & Fase & Data & Hora & Local & $\begin{array}{c}\text { Número } \\
\text { de fotos }\end{array}$ & $\begin{array}{c}\text { Distância } \\
{[\mathrm{m}]}\end{array}$ & $\begin{array}{c}\text { Altitude } \\
\text { máx [m] }]\end{array}$ & $\begin{array}{c}\text { Duração } \\
{[\mathrm{min}]}\end{array}$ \\
\hline 1 & Teste & $05 / 08 / 2018$ & $11: 14$ & IIT-UPE & 2 & 20 & 5 & 15 \\
\hline 2 & Teste & $05 / 08 / 2018$ & $11: 40$ & IIT-UPE & 35 & 8 & 16 & 12 \\
\hline 3 & Teste & $07 / 08 / 2018$ & $09: 52$ & IIT-UPE & 108 & 4 & 24 & 27 \\
\hline 4 & Teste & $07 / 08 / 2018$ & $11: 31$ & IIT-UPE & 95 & 4 & 35 & 28 \\
\hline 5 & Teste & $13 / 08 / 2018$ & $14: 15$ & IIT-UPE & 42 & 6 & 92 & 8 \\
\hline 6 & Teste & $13 / 08 / 2018$ & $14: 27$ & IIT-UPE & 67 & 8 & 35 & 19 \\
\hline 7 & Teste & $13 / 08 / 2018$ & $15: 50$ & IIT-UPE & 87 & 8 & 36 & 25 \\
\hline 8 & Teste & $30 / 08 / 2018$ & $15: 25$ & IIT-UPE & 35 & 50 & 120 & 24 \\
\hline
\end{tabular}

As imagens obtidas através dos voos feitos no Parqtel foram analisadas de duas formas. A primeira forma foi olhando imagem por imagem com o intuito de verificar algum tipo de manifestação patológica. Esse método se mostrou viável para identificação de vários tipos de manifestações patológicas, como: eflorescência, bolor e fissura, representada pelas figuras 7,8 e 9 . 


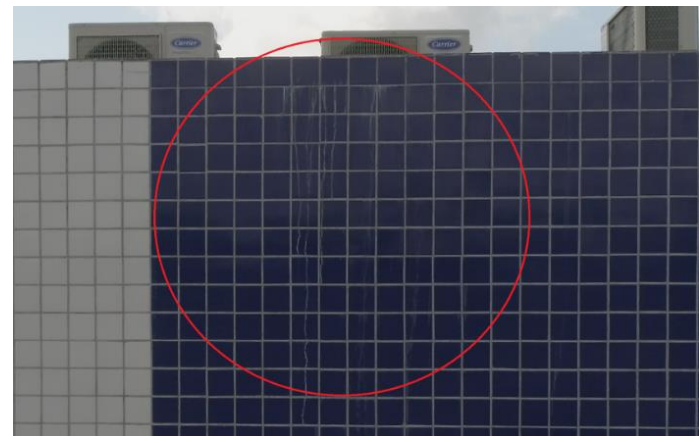

Figura 7: Eflorescência

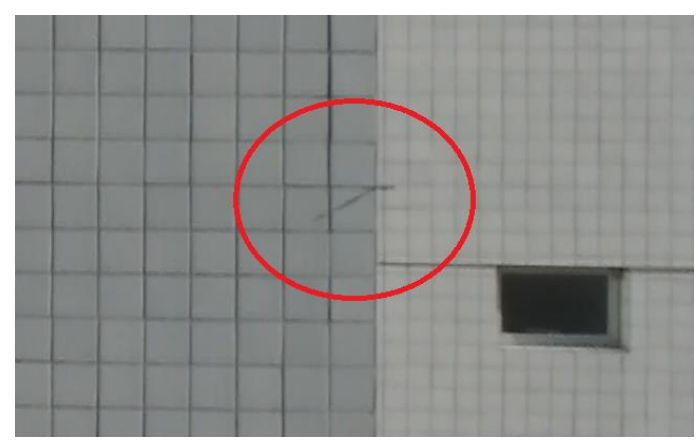

Figura 8: Fissura

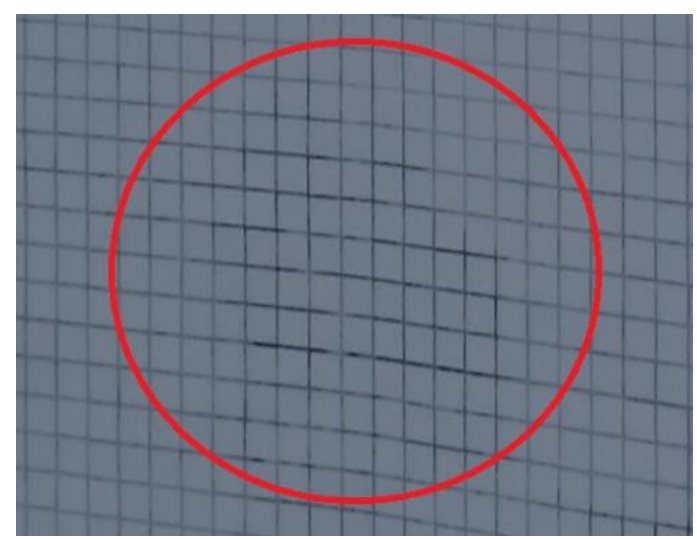

Figura 9: Bolor

A segunda forma para análise de manifestações foi utilizando o software PhotoScan, onde as imagens eram selecionadas e o conjunto de algoritmo realizava um reconhecimento dos pontos georreferenciados. A partir da inserção das imagens coletadas em campo é possível criar uma nuvem densificada de pontos que começa a dar modelo para o prédio. Após isso é feito a texturização da modelagem 3D até obter o produto final, conforme a figura 10. É possível também gerar um ortomosaico de cada fachada, representado na figura 11.

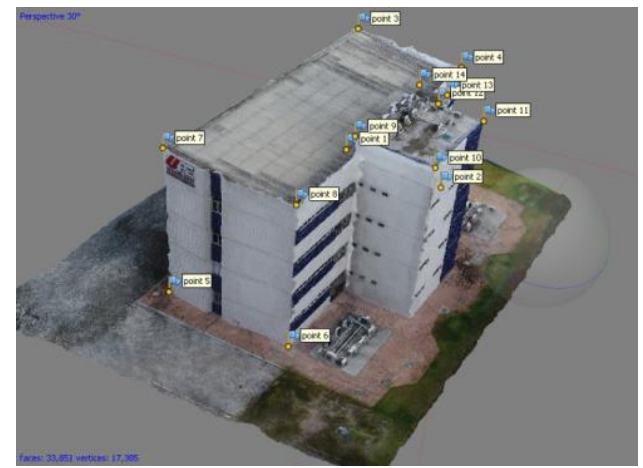

Figura 10: Modelo 3D PhotoScan 


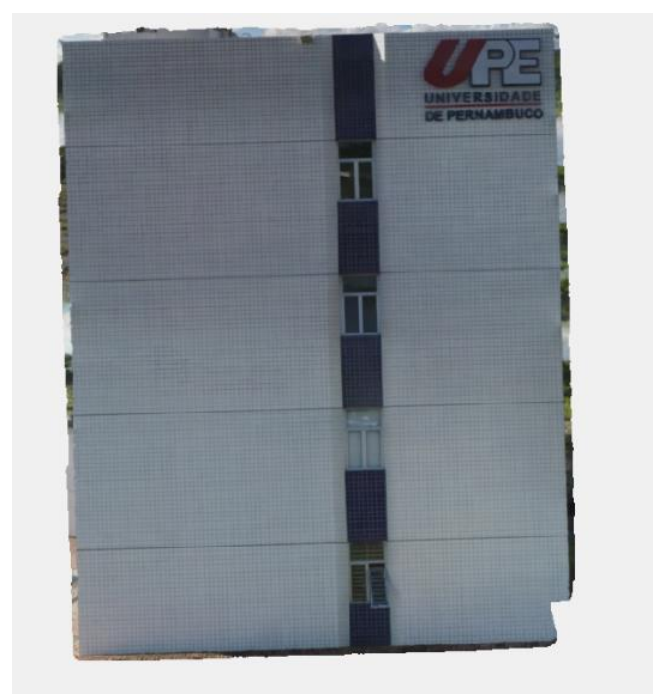

Figura 11: Ortomosaico

\section{CONCLUSÃO}

A inspeção de fachadas associada ao drone mostrou-se como uma técnica efetiva para análise de inspeção de manifestações patológicas em fachadas, principalmente pela possibilidade de acesso a diferentes locais de forma rápida, análise de áreas maiores ao invés de pontos específicos, e sem necessitar de muito espaço para locação do equipamento, tendo em vista que os drones possuem tamanho relativamente pequeno. Além disso, é importante salientar que a técnica é mais segura comparada aos métodos usuais, já que o VANT que é o meio para análise, eliminando os riscos que o ser humano estaria submetido. O método também mostrou ter uma melhor eficiência quanto a velocidade, já que, dependendo do tamanho do prédio e equipamento utilizado, a inspeção pode ser feita em apenas de um voo.

Utilizando a câmera fotográfica não é possível identificar todos os tipos de manifestações patológicas presentes em uma fachada, como é o caso de infiltrações. Para isso, é possível acoplar uma câmera termográfica ao drone para estudo desse tipo de manifestação patológica.

A pesquisa exploratória de campo serviu para caracterizar o panorama atual das empresas de drone na Região Metropolitana do Recife - RMR/PE. A partir dos resultados foi possível identificar padrões relacionados ao planejamento de voo e a classificação dos drones, o que ajudou no estudo do assunto para esse artigo.

Algumas barreiras relacionadas à utilização dos modelos 3D foram identificadas, como a presença de alguns defeitos visuais, ocasionados principalmente por falta de pontos suficientes para reconhecimento do programa, devido a falta de mais imagens ou falha no planejamento do voo, presença de fortes ventos e alternância de luminosidade. Entretanto, de acordo com a percepção dos usuários, as falhas visuais identificadas não representaram grande impacto na dificuldade de utilização dos modelos 3D para os fins propostos.

\section{AGRADECIMENTOS}

Gostaria de agradecer ao Professor Dr. Alberto Casado por todos os ensinamentos durante a pesquisa. Agradecer também ao mestrando e amigo Ramiro Ballesteros. Agradecer ao Programa de Fortalecimento Acadêmico (PFA) da Univerdade de Pernambuco (UPE) pela bolsa recebida durante o ano de desenvolvimento da pesquisa.

\section{REFERÊNCIAS}

AGOSTINHO, S. L. Inspeção e Monitorização de Estruturas em Engenharia Civil - Utilização de UAV na Inspeção e Monitorização.2012. 105 f. Dissertação (Mestrado em Engenharia), Universidade da Madeira, Madeira, Portugal, 2012. 
ÁlVARES, J.; COSTA, D. B.; MELO, R. R. S.; BELLO, A. Estudo exploratório de mapeamento 3D de canteiros de obras utilizando veículos aéreos não tripulados. In: XVI ENTAC, 1., 2016. São Paulo. Anais... São Paulo, 2016. ALVES, K. C. C. O processo de assistência técnica de empresas de construção: estudos de caso. Recife, 2016. 117p. Dissertação - Escola Politécnica de Pernambuco.

ANAC. Agência Nacional de Aviação Civil. Requisitos gerais para aeronaves não tripuladas de uso civil - RBACE no 94. Resolução n. 419, 2 de maio de 2017. Brasília, 2017.

CAMPOS, M. Queixas contra imóveis novos crescem no Procon. Disponível em: < http://gazetaonline.globo.com/_conteudo/2011/05/noticias/a_gazeta/economia/862139-queixas-contra-imoveis-novoscrescem-no-procon.html>. Acesso em 12 set. 2013.

CEOTTO, L.H; BANDUK, R.C.; NAKAKURA. Revestimentos de argamassas: boas práticas em projeto, execução e avaliação. Porto Alegre: HABITARE/ABCP, 2005.

CONSTRUBUSINESS 2016. Investir com responsabilidade. Disponível em: <http://www.fiesp.com.br/?temas=observatorio-da-construcao>. Acesso em: 12 mar. 2017.

COSTA E SILVA, A. J. Método para gestão das atividades de manutenção de revestimentos de fachada. São Paulo, 2008. 221p. Tese (Doutorado) - Escola Politécnica, Universidade de São Paulo.

EMELIANOV, S.; BULGAKOW, A., SAYFEDDINE, D. Aerial laser inspection of buildings facades using quadrotor. Creative Construction Conference, Feb. 2014. Prague. p. 6.

FIGUEIREDO JÚNIOR, G. J. Patologias em revestimentos de fachadas - Diagnóstico, prevenção e causas. 2017. 92f. Trabalho de conclusão de curso - Universidade Federal de Minas Gerais, Belo Horizonte, 2017.

GOMES, N. G. U. Integração entre planejamento e contratações na construção civil. Téchne: PINI, 2017.

LICHTENSTEIN, N. B. Patologia das Construções: procedimento para formulação do diagnóstico de falhas e definição de conduta adequada à recuperação de edificações: São Paulo: Escola Politécnica da USP, 1985. Dissertação (Mestrado em Engenharia Civil) - Universidade de São Paulo, 1985.

LORDSLEEM JÚNIOR, Alberto Casado. Sistemas de recuperação de fissuras da alvenaria de vedação: avaliação da capacidade de deformação. 1997. 174 f. Dissertação (Mestrado em Engenharia Civil) - Departamento de Engenharia de Construção Civil, Universidade de São Paulo, São Paulo, 1997.

MELO, R. R. S.; COSTA, D. B. Uso de veículo aéreo não tripulado (VANT) para inspeção de logística em canteiros de obra. SIBRAGEC-ELAGEC, outubro 2015. São Carlos, p. 674. Anais...

NASCIMENTO, M. L. M. Utilização de drone e termografia na detecção de manifestações patológicas em edificações. Brasília, 2014. 21p. Monografia (Graduação) - Universidade Católica de Brasília.

PINHO, S. A. C. Desenvolvimento de programa de indicadores de desempenho para tecnologias construtivas à base de cimento: perdas, consumo e produtividade. Recife, 2013. 268p. Dissertação (Mestrado) - Escola Politécnica, Universidade de Pernambuco.

PROCON - Fundação de Proteção e Defesa do Consumidor. Cadastro de reclamações fundamentadas, 2011: kit de imprensa. São Paulo: 2012.

RIBEIRO, D.V. Corrosão em estruturas de concreto armado: teoria, controle e métodos de análises. Rio de Janeiro: Elsevier, 2014.

THOMAZ, E. Trincas em edifícios. São Paulo, IPT/EPUSP/PINI, 1989. 